\title{
SERIAL RESONANT CONVERTER AND LOAD COIL FOR HIGH FREQUENCY HEATING
}

The paper deals with the design of the voltage source half-bridge resonant inverter with the specific load coil, which is suitable for induction heating applications. To ensure the correct functionality and right frequency range of the self-resonant converter, the parameters as inductance, self-capacitance and resistance of the coil have to be obtained. Parameters of the coil are estimated by utilizing software that uses finite elements method of solution. In order to estimate the accuracy of model, the experimental verifications were taken. For this purpose, the spiral coil made on printed circuit board was created and its parameters were calculated, simulated and compared with measurement results.

Keywords: Induction heating, induction coil, resonant converter, finite elements method.

\section{Introduction}

Induction heating $(\mathrm{IH})$ is widely used in many industrial applications including melting, heating prior to metalworking hardening, during manufacturing of semiconductors (doping, refining) and in domestic applications such as induction cookers. Electromagnetic induction is a method of heating electrically conductive materials such as metals. Such phenomenon relies on two mechanisms of energy dissipation. These are losses due to Joule heating and losses associated with magnetic hysteresis. First of these, is primary mechanism of heat generation in electrically conductive nonmagnetic materials (aluminum, copper, steels above Curie temperature). Electrical currents or eddy currents which are internally induced in material dissipate the energy and bring about the heat. Second mechanism is present in magnetic materials. The explanation of hysteresis losses states that heat generation is caused by friction between molecules, or so called magnetic dipoles, when ferromagnetic metals are first magnetized in one direction and then in the other. The energy needed to turn the dipoles is dissipated as heat.

The induction heating system (Fig. 1) consists of a source of alternating current, an induction coil and load which is eddy current based heating material.

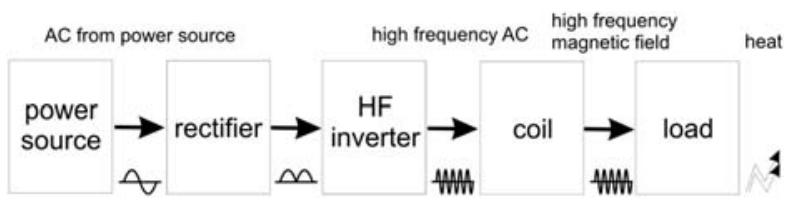

Fig. 1 Induction heating system
The coil is connected to the power supply. The flow of AC current through the coil generates the alternating magnetic field which cuts through the workpiece and thus generates the heat. Generation of heat increases with frequency and amplitude of AC current. The more frequency increases, the more are eddy currents located near the surface of workpiece. The common characteristic of the induction heating applications are high efficiency, quick heating, energy savings, ease of automation, quiet, clean and safe working conditions [1], [2].

\section{High frequency converters}

IH system as stated before consists of source of AC current coil and the workpiece to be heated. There are various high frequency inverter circuit topologies, such as: full bridge, half bridge, single-ended push-pull, center tap push-pull and boost half bridge. Most of the IH systems are constructed as resonant inverters operating at high frequencies. Of these, the voltage source type ZCS (zero current switching), ZVS (zero voltage switching), ZVZCS (zero voltage - zero current switching), SEPP (Single-Ended Push Pull) resonant and quasi-resonant hybrid high frequency inverter has unique features; such as simple configuration, high efficiency and wide soft commutation range [3].

Increasing the switching frequencies improves the performance and power density of the inverter. However, with increasing the switching frequencies some disadvantages appear due to the high commutation losses in the switching and appearance of electromagnetic interference. These factors occur mainly in inverter topologies that use the full-bridge inverter configuration. The use of resonant converter which offers the interesting option for an application requires previous specification [4], [5], [6].

\footnotetext{
* Matus Ocilka ${ }^{1}$, Dobroslav Kovac ${ }^{1}$, Irena Kovacova ${ }^{1}$, Jan Perdulak ${ }^{1}$, Andrii Gladyr ${ }^{2}$, Dmytro Mamchur ${ }^{2}$, Iurii Zachepa ${ }^{2}$, Tibor Vince ${ }^{1}$, Jan Molnar ${ }^{1}$

${ }^{1}$ Address: Department of Theoretical Electrical Engineering and Electrical Measurement, Technical University of Kosice, Slovakia,

E-mail: matus.ocilka@tuke.sk

${ }^{2}$ Address: Department of Automatic Management and Electrical Drive, Kremenchuk Mykhailo Ostrohradskyi National University, Ukraine
} 
Voltage source half-bridge resonant inverter

The use of full-bridge resonant converter brings some disadvantages due to high switching losses and electromagnetic interference. Moreover, the configuration and control algorithm of such a converter is more complicated than, for example, in halfbridge topology. For our purpose the voltage source half-bridge resonant converter topology is used. The converter topology is depicted in Fig. 2 and consists of two switches $\left(\mathrm{S}_{1}, \mathrm{~S}_{2}\right)$, two antiparallel diodes and load which is a working coil that serves as a resonant circuit.

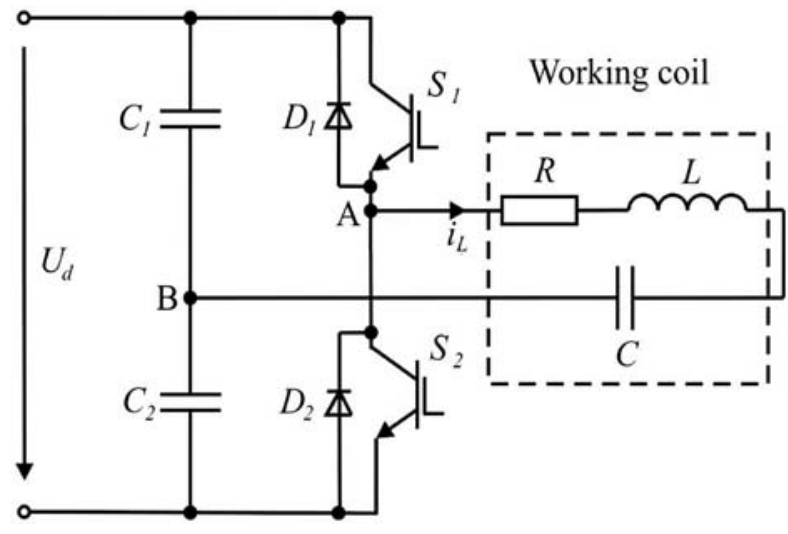

Fig. 2 Half-bridge series resonant inverter

The parameters of working coil such as inductance, self-capacitance and resistance have to be known because the switching frequency of converter has to be set as close as possible to resonant frequency of the coil/circuit. Since the self-capacitance of coils is in general very small, the resonant frequencies lie in a range of $\mathrm{MHz}$. The desired resonant frequency is commonly set by adding tuning capacitor into the circuit. In our case, no tuning capacitance is added to the resonant circuit, thus the careful attention in designing the coil has to be taken to bring the resonant frequency of the coil in a range of hundreds of $\mathrm{kHz}$. For obtaining these parameters for given coil designs the finite elements method of solution is used.

\section{Finite elements method of solution}

For calculation of inductance and self-capacitance of coils there are several analytic approaches and models. Due to the complex nature of the approximations, where the coil's geometry, mutual inductance or self inductance has to be considered, the analytic approach can be overly difficult. High accuracy and precision during design stage is essential, because when printed on circuit board, the inductor can't be changed anymore. Thus the using of finite element method can be a good alternative of solving such problems and can give us information about parameters of the coil in some range and can provide us with some initial conditions in designing such devices [7], [8].
For our purpose the software package called COMSOL Multiphysics was used. It is a software that solves systems of coupled three-dimensional partial differential equations. The COMSOL Multiphysics engineering simulation software environment facilitates all steps in the modeling process - defining geometry, meshing, specifying physics, solving, and then visualizing results. Model setup is quick thanks to a number of predefined physics interfaces for applications ranging from fluid flow and heat transfer to structural mechanics and electromagnetic analyses. Material properties, source terms and boundary conditions can all be arbitrary functions of the dependent variables. It can model the complex problems, geometries or material properties in $2 \mathrm{D}$ or $3 \mathrm{D}$ representation for better understanding of some phenomena. In this case, COMSOL Multiphysics solves the magnetic and electric fields created by the coil that is placed in electrical circuit and calculates the inductance, resistance and capacitance of the coil.

\section{Simulation models and experimental verification}

The spiral coil is a spiral - shaped formation made of copper. This copper spiral is electrically conductive showing electrical resistance for the direct current. For the alternating current the inductor shows, apart from electrical resistance, also a property called inductance, which describes the capability of producing a magnetic field around the inductor. From the electrical point of view the inductance acts as complex impedance being proportional to the frequency of alternating current. The ideal inductor shows only the inductance while its electrical resistance is negligible.

\section{The coil geometry}

The proposed double-layer coil is shown in Fig. 3.

The above mentioned coil is physically described by the following parameters: $d_{i n}$ as the inner diameter [mm], $d_{\text {out }}$ is outer diameter [mm], $s$ expresses the spacing between turns [mm], $w$ is the width of conductor [mm], $h$ is height of conductor [mm] and $d[\mathrm{~mm}]$ represents the distance of both spirals.

Inductance of the coil - analytic solution

Calculation of inductance value of one layer of such coil can be done using the following equation [9]:

$$
L=\frac{\mu_{0} N^{2} d_{a v g} C_{1}}{2}\left(\left(\ln \frac{C_{2}}{\rho}\right)+C_{3} \rho+C_{4} \rho^{2}\right) .
$$

Where $\rho$ is the fill ratio:

$$
\rho=\frac{d_{\text {out }}-d_{\text {in }}}{d_{\text {out }}+d_{\text {in }}} .
$$

And $d_{\text {avg }}$ is average diameter:

$$
d_{\text {avg }}=\frac{d_{\text {out }}-d_{\text {in }}}{2} \text {. }
$$

Constants $C_{1}-C_{4}$ are shape dependent constants and their values according to [9], are $C_{1}=1.00, C_{2}=2.46, C_{3}=0.00$, $C_{4}=0.20$, and $\mu_{0}=4 \pi \times 10^{-7} \mathrm{H} \cdot \mathrm{m}^{-1}$. The inductance of doublelayer coil is given by the following equation 


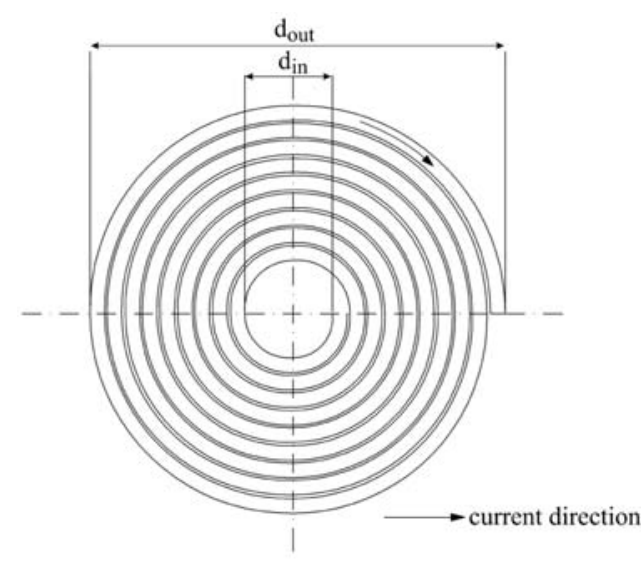

a)

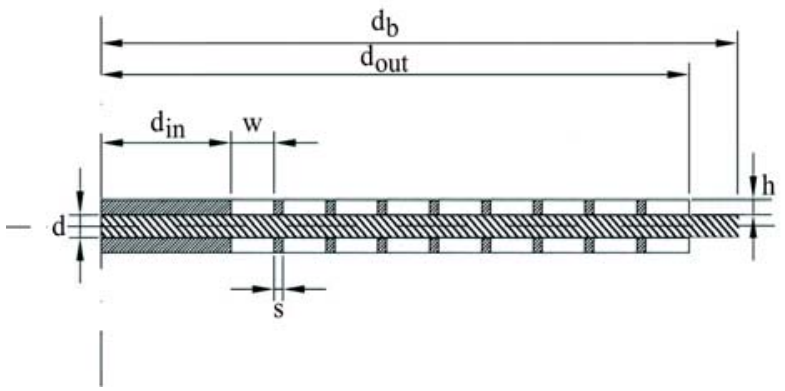

b)

Fig. 3 Designed double-layer spiral coil a) top view b) side view

$$
L_{\text {tot }}=L_{1}+L_{2} \pm 2 M \text {. }
$$

Where $L_{1}$ and $L_{2}$ are the self-inductances of both coils and $M$ is the mutual inductance between coils. Mutual inductance is difficult to calculate because it depends on many factors as follows: distance between coils, geometry, and mutual geometry of particular conductors. The inductance increases or decreases total inductance of the coil depending on currents directions passing through the coils. In our case the currents of both coils pass through coils in the same direction. The mutual inductance can be determined as:

$$
M=K_{c} \sqrt{L_{1} L_{2}} .
$$

Where $K_{c}$ is the coupling factor and its value must lie in interval $\langle 0 ; 1\rangle$. The coupling factor can be determined using the following formula [9]:
Where $x=d$ is the mutual distance of both coils, constants $A$, $B, C, D$ according to [9], are:

$$
A=0.184, B=-0.525, C=1.038, D=1.001 \text {. }
$$

Inductance of the coil - simulation model

The model of coil is shown in Fig. 4a) and it is modeled in COMSOL Multiphysics in AC/DC module. The model is created in 2D axisymmetric space dimension and the coil is surrounded with an air domain and its boundary serves as magnetic insulation [10].

This model is not exact spiral geometry and it was adapted for solving in 2D axisymmetric space dimension because spiral-shaped formation of the coil is not axisymmetric. One layer of the coil is created from 9 rings as depicted in Fig. 4b).

$$
K_{c}=\frac{N^{2}}{0.64 \cdot\left(A x^{3}+B x^{2}+C x+D\right) \cdot\left(1.67 N^{2}-5,84 N+65\right)} .
$$

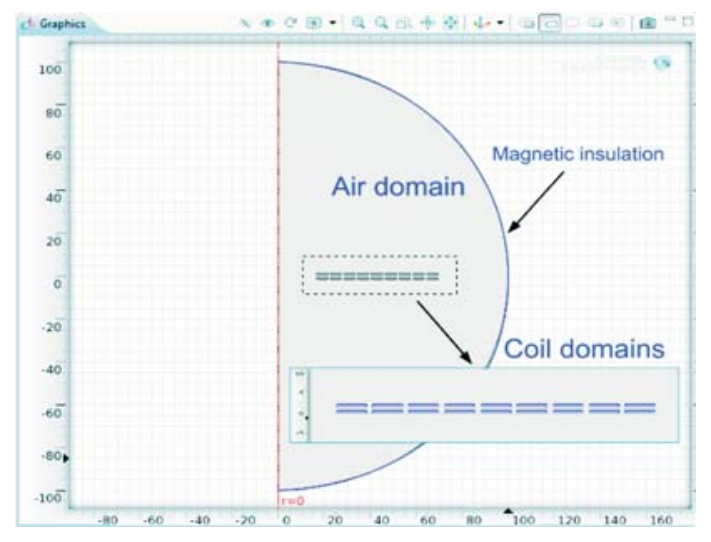

a)

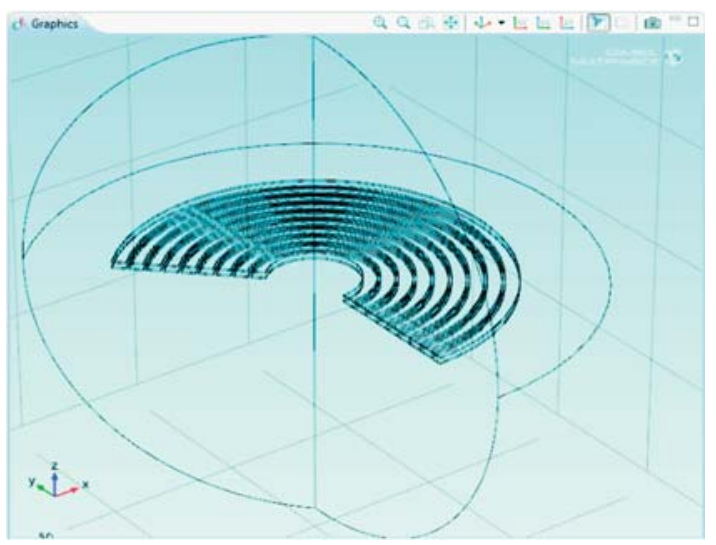

b)

Fig. 4 Double-layer coil in COMSOL a) model of the coil b) model of the coil in $3 D$ 
The parameters used in calculation and simulations are in Table. 1.

Parameters used in simulation for double-layer coil

Tab. 1

\begin{tabular}{|c|c|}
\hline Number of turns: & $\mathrm{N}=9$ \\
\hline Outer diameter: & dout $=142 \mathrm{~mm}$ \\
\hline Inner diameter: & $\mathrm{din}=30 \mathrm{~mm}$ \\
\hline Width of conductor: & $\mathrm{W}=5 \mathrm{~mm}$ \\
\hline Spacing between turns: & $\mathrm{s}=1 \mathrm{~mm}$ \\
\hline Height of conductor: & $\mathrm{h}=0.8 \mathrm{~mm}$ \\
\hline Distance of coils: & $\mathrm{d}=1.5 \mathrm{~mm}$ \\
\hline
\end{tabular}

\section{Analytic results}

The inductance of one coil using (1) is $L_{1}=6.19 \mu \mathrm{H}$. Both coils have the same geometry, so the inductance of second coil must be the same $L_{2}=6.19 \mu \mathrm{H}$. Using (4) we can calculate the total inductance of double-layer coil. The coupling factor according to (6) is $K_{c}=0.855$ and mutual inductance (5) is $M=5.29 \mu \mathrm{H}$. Then the total inductance using analytic approach is $L_{\text {tot }}=22.90 \mu \mathrm{H}$.

\section{Simulation results}

For simulation the model shown in Fig. 4a) was used. Copper with conductivity $\sigma=5.997 \times 10^{7} \mathrm{~S} . \mathrm{m}^{-1}$ was selected as the material for coil. The coil was driven by current $I_{0}=20 \mathrm{~A}$, the model was calculated in a frequency domain with $f=60 \mathrm{~Hz}$. Mesh is a very important parameter of simulation. Proper size and shape of mesh elements influence the accuracy of solution; the finer the mesh elements, the better the solution accuracy. It is very important to find optimal number of elements. In our case the triangular mesh of 288500 elements with maximum size of $2 \mathrm{~mm}$ was created.

Inductance of coil solved by the software is $L=24.04 \mu \mathrm{H}$. Fig. 5 shows magnetic flux density. The magnetic field is strongest

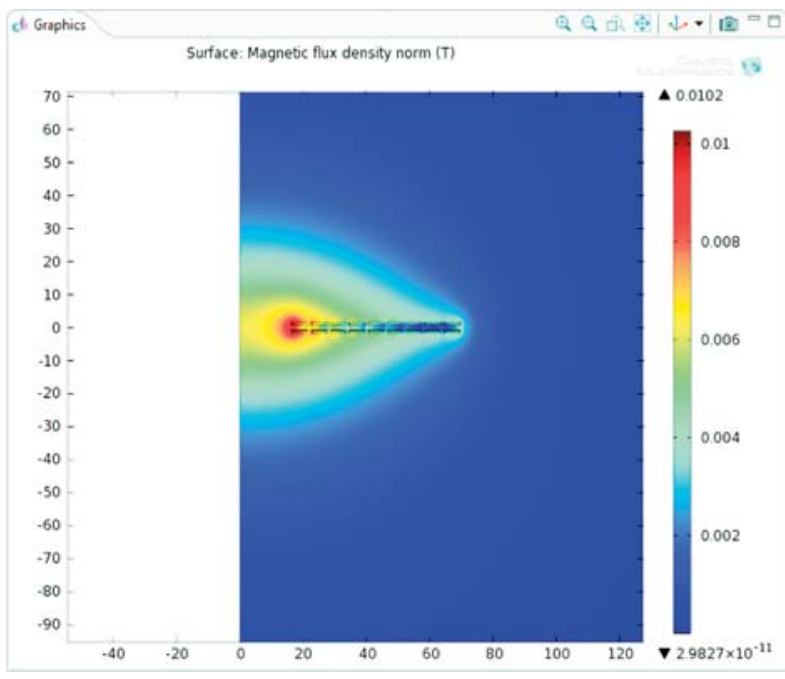

Fig. 5 Magnetic flux density

in the middle of the coil and decreases with increasing radius of the coil.

\section{Experimental measurements}

The experimental measurements were taken with a coil made on a printed circuit board. The values of inductance are listed in Table 2.

Experimental measurements of double-layer coil

Tab. 2

\begin{tabular}{|c|c|c|c|c|c|}
\hline Frequency & 60 & $5 \mathrm{k}$ & $100 \mathrm{k}$ & $200 \mathrm{k}$ & {$[\mathrm{Hz}]$} \\
\hline $\mathrm{L}$ & 25.0 & 25.0 & 24.5 & 24.0 & {$[\mu \mathrm{H}]$} \\
\hline
\end{tabular}

It can be seen that the difference between the simulation model and experimental measurements at frequency $f=60 \mathrm{~Hz}$ is $3.84 \%$

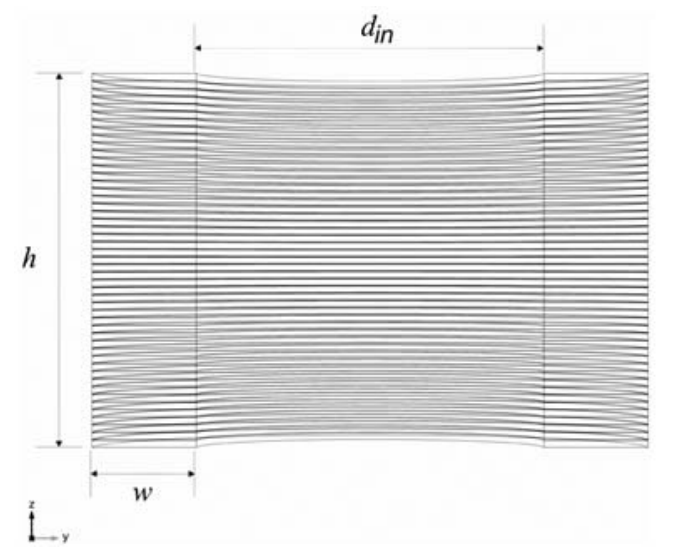

a)

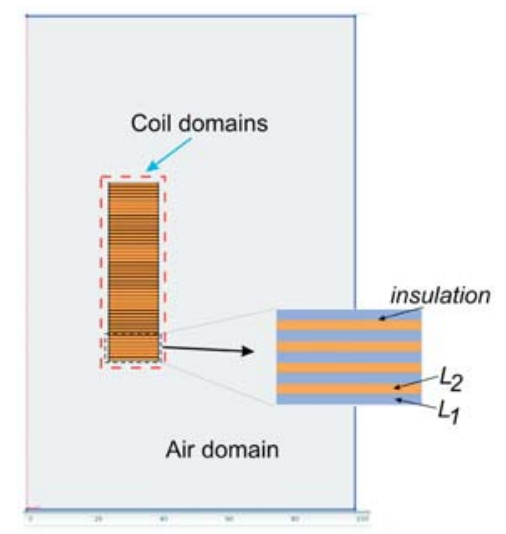

b)

Fig. 6 Working coil for IH application a) designed coil b) model of the coil in COMSOL 
and difference between analytic and experimental measurements is $8.4 \%$.

\section{Load coil for converter}

The proposed coil for IH heating application is illustrated in Fig. 6a). The coil is made of two coils connected in series. The outer coil has 25 turns and between these turns the turns of the second coil are inserted. This coil has 24 turns. The thickness of insulation between each turn is $d_{i}=0.1 \mathrm{~mm}$. This insulation serves as dielectric material.

Since the coil serves as a resonant circuit and no additional tuning capacitor is used the self-resonant frequency of the coil can be controlled by setting the number of turns of the coil, or by changing the cross section area of conductor, or distance between particular turns. Such circuit is shown in Fig. 7.

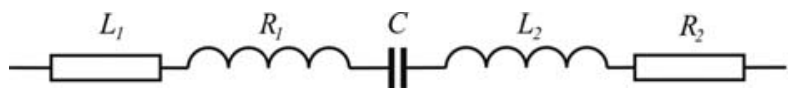

Fig. 7 Circuit of proposed coil

To achieve the desired resonant frequency the parameters such as inductance and resistance of both coils and the capacitance of the whole system have to be calculated. For this purpose the above mentioned approach for a double-layer coil can be used because the difference between the simulation and experimental measurement is $3.84 \%$.

The simulation model of this coil is depicted in Fig. 6b). In order to solve inductance in COMSOL, the magnetic fields toolbox (MF) was selected. Calculation was made in a frequency domain with frequency set at $f=50 \mathrm{kHz}$ and the coils were driven by current $I=10 \mathrm{~A}$. The simulation results for magnetic field are shown in Fig. 8.

Figures 8 shows the magnetic field created by current passing through both coil. Again, it can be seen that the magnetic field is strongest in the middle of the coil. The calculated parameters (at $f=50 \mathrm{kHz}$ ) of this coil are listed in Table 3 .

Calculated parameters of designed coil

Tab. 3

\begin{tabular}{|l|c|}
\hline Inductance of first coil & $L_{1}=35.5627 \mu \mathrm{H}$ \\
\hline Resistance of first coil & $R_{1}=393.74 \mathrm{~m} \Omega$ \\
\hline Inductance of second coil & $L_{2}=34.4492 \mu \mathrm{H}$ \\
\hline Resistance of second coil & $R_{2}=416.55 \mathrm{~m} \Omega$ \\
\hline Capacitance of coil & $C=30.1397 \mathrm{nF}$ \\
\hline
\end{tabular}

The resonant frequency of this circuit is $f_{0}=109827 \mathrm{~Hz}$. The switching frequency of converter has to be set equal to resonant frequency of the circuit.

Simulation of converter

The theoretical waveforms of this topology that are obtained in PSPICE are shown in Fig. 9.

The switching frequency of converter was approximately $f_{s}=$ $=110 \mathrm{kHz}$. The control algorithm adjusts the switching of transistors when load current crosses zero value. It can be seen that the switching losses occur on the switches because the control of converter delays the turn on/off of transistors by approximately 2-3 $\mu$ s. This problem can be removed by switching the transistors before the load current reaches zero.

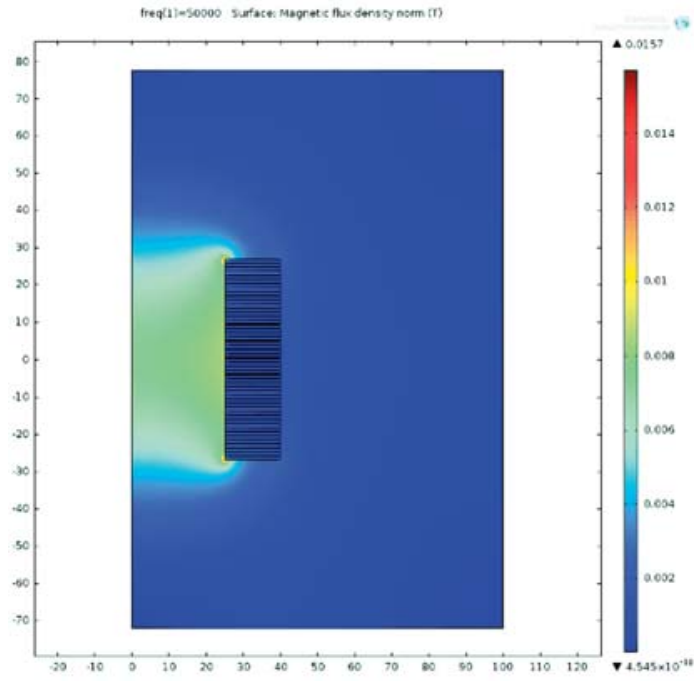

a)

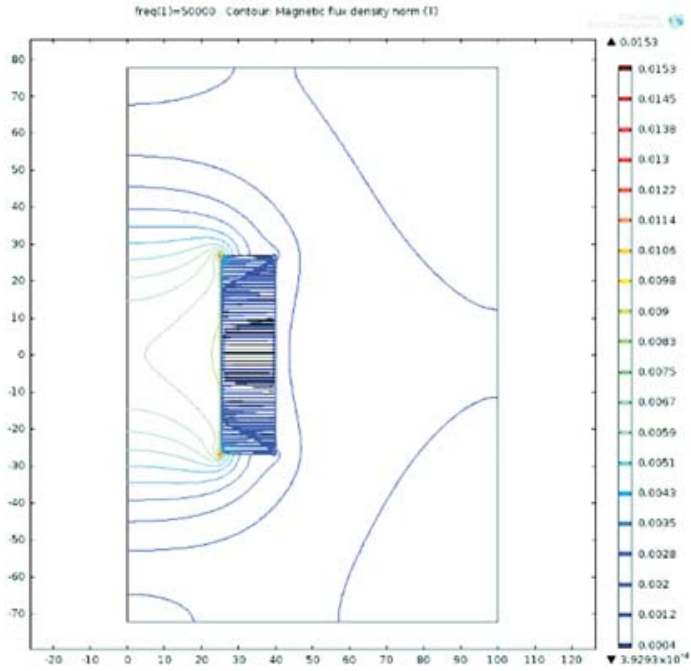

b)

Fig. 8 Magnetic flux density [T] a) surface b) contour 


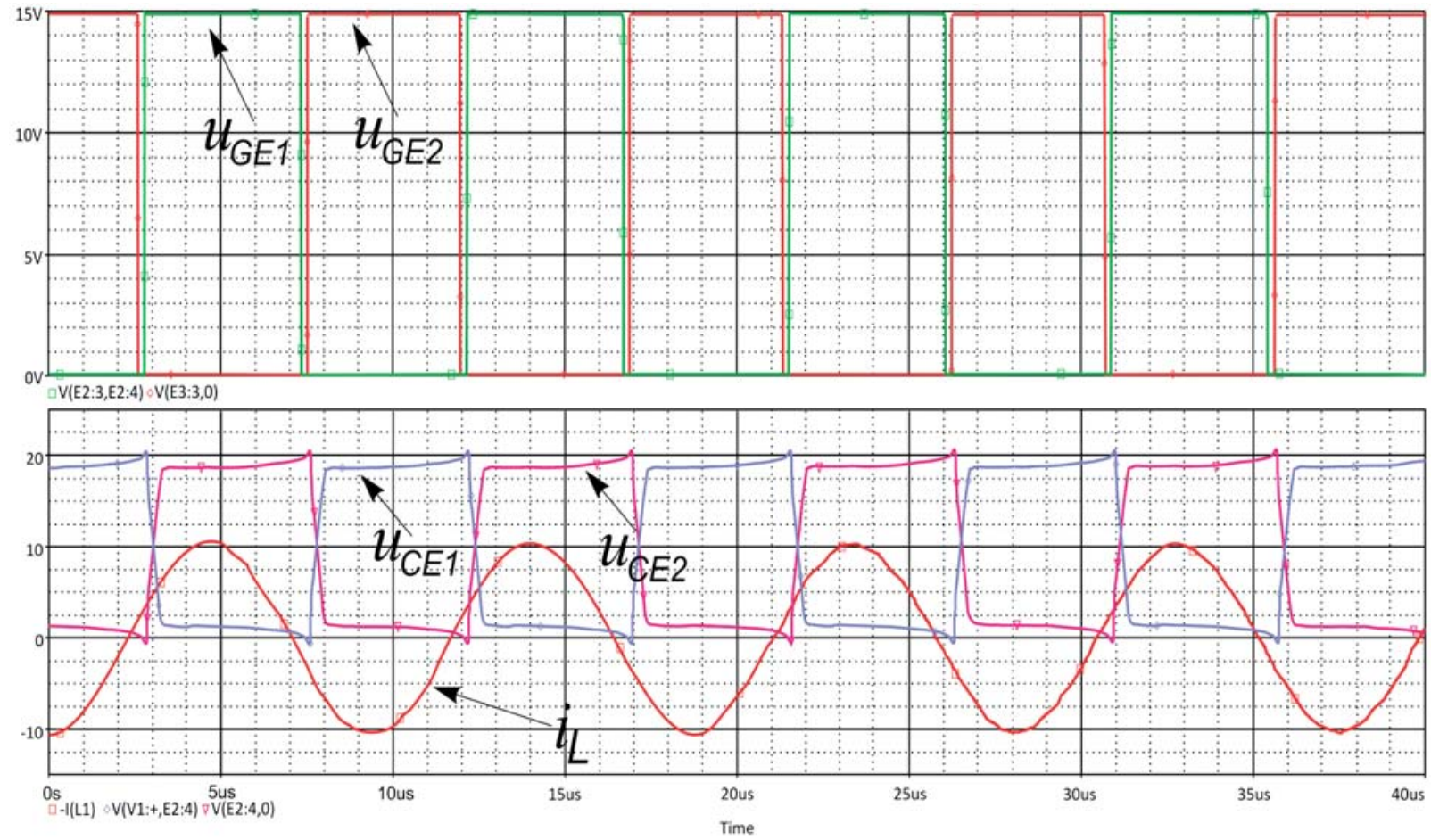

Fig. 9 Waveforms of converter in PSPICE

\section{Conclusion}

In the paper the half bridge series resonant converter and load coil for this converter were presented. The converter topology is simple configuration, ensures suitable control and offers the best overall performance with respect to converter utilization. In the paper the simulation waveforms of proposed converter were presented. With a suitable algorithm of control, the switching losses on transistor can be reduced and thus the efficiency of inverter can be increased. In the next part the load coil for the inverter was presented. The coil serves as a resonant circuit for converter. First, the verification model of spiral coil was created to verify the model accuracy with experimental measurement on a real double-layer coil built on a printed circuit board. Using this simulation the model of load coil for induction heating was created. The simulation provided us with parameters such as inductance, resistance and selfcapacitance. The parameters were then used in simulation of the converter as load. It can be seen that the self-resonant frequency of coil was brought to approximately $100 \mathrm{kHz}$. The future work will be focused on building the system utilizing such designed coil and converter.

\section{Acknowledgment}

The paper has been prepared under the support of Slovak grant projects KEGA No. 005TUKE-4/2012.

\section{References}

[1] ZIN, S., SEMIATIN, S. L.: Elements of Induction Heating: Design, Control, and Applications, ASM International, 1988

[2] DOLEZEL, I., ULRYCH, B.: Induction Heating of Very Thin Metal Plates Modelled by Electric Vector Potential. Communications - Scientific Letters of the University of Zilina, No. 2A, 2011, pp. 19-24.

[3] MIURA, Y., HASHEM, M. H., HIRAKI, E., YASURI, K., IWAI, T., AHMED, N. A., LEE, H. W., NAKAOKA, M.: A New Zero Current Switching Duty Cycle Controlled High Frequency Multi Resonant Inverter for Consumer Power Applications, Electrical Machines and Systems, ICEMS 2005, Sept. 2005, pp. 1112-1117.

[4] KOVACOVA, I., KOVAC, D., VINCE, T.: Electromagnetic Compatibility, Published by TU FEI, Kosice, p. 138, 2009, ISBN 97880-553-0150-1.

[5] KOVAC, D., KOVACOVA, I., MOLNAR, J.: Electromagnetic Compatibility - Measurement, Published by TU, FEI, pp. 72, 2009, ISBN 978-80-553-0151-8. 


\section{COMMNICOIIONS}

[6] SPANIK, P., KANDRAC, J., FRIVALDSKY, M., DRGONA, P.: Verification of Operation Modes of Designed LLC Resonant Converter, Communications - Scientific Letters of the University of Zilina, No. 2A, 2011, pp. 67-73

[7] Paese, E., Geier, M., Pacheco, J. L., Homrich, R. P., Ortiz, J. C. S.: Mathematical Modeling of an Electromagnetic Forming System with Flat Spiral Coils as Actuator, $4^{\text {th }}$ Intern. Conference of High Speed forming, 2010, pp. 219-228.

[8] TANG, W., ZHU, Y., CHOW, Y. L.: Inductance Calculation of Spiral Inductors in Different Shapes, Proc. of Microwave Conference, 2005. APMC 2005. Asia-Pacific, vol. 5, pp. 3, 4-7, Dec. 2005.

[9] ZHAO, J.: A New Calculation for Designing Multilayer Planar Spiral Inductors, Electronics Design, Strategy, News, July 2010.

[10] KOVAC, D., OCILKA, M., VANSAC, M.: Calculating of Inductance of Spiral Coil Using COMSOL Multiphysics: ESMO 2012, Kremenchuk Mykhailo Ostrohradskyi National University, Ukraine, pp. 89-90, ISSN 2079-5106. 